Documentation et bibliothèques

DOCUMENTATION BIBLIOTHËQUES

\title{
L'utilité des services de support documentaire dans les cégeps du Québec : l'opinion des enseignants
}

\section{Daniel Morin}

Volume 30, numéro 4, octobre-décembre 1984

URI : https://id.erudit.org/iderudit/1053534ar

DOI : https://doi.org/10.7202/1053534ar

Aller au sommaire du numéro

Éditeur(s)

Association pour l'avancement des sciences et des techniques de la

documentation (ASTED)

ISSN

0315-2340 (imprimé)

2291-8949 (numérique)

Découvrir la revue

Citer cet article

Morin, D. (1984). L'utilité des services de support documentaire dans les cégeps du Québec : l'opinion des enseignants. Documentation et bibliothèques, 30(4),

137-140. https://doi.org/10.7202/1053534ar

Tous droits réservés (c) Association pour l'avancement des sciences et des techniques de la documentation (ASTED), 1984
Ce document est protégé par la loi sur le droit d'auteur. L’utilisation des services d’Érudit (y compris la reproduction) est assujettie à sa politique d'utilisation que vous pouvez consulter en ligne.

https://apropos.erudit.org/fr/usagers/politique-dutilisation/ 


\section{L'utilité des services de support documentaire dans les cégeps du Québec: I'opinion des enseignants}

Une enquête a été menée au printemps 1983 auprès d'enseignants de collèges d'enseignement général et professionnel (cégep) sur l'utilité de différents services de support documentaire à l'enseignement. Cette enquête se situe à l'intérieur de la problématique des relations entre enseignants et spécialistes en ressources documentaires dans l'enseignement (SMTE' dans le texte).

D'emblée, on peut se demander pourquoi il faille s'intéresser à l'opinion des enseignants. II nous est apparu que, sous le régime pédagogique actuel, l'enseignant constitue un intervenant majeur, car il détermine une grande part des conditions dans lesquelles l'étudiant sera appelé à effectuer ses apprentissages. Aussi le comportement des enseignants, en ce qui a trait à l'exploitation des ressources documentaires, a-t-il des conséquences directes sur le degré d'intégration de ces ressources aux méthodes d'enseignement. On en déduit évidemment qu'il est essentiel que la relation professionnelle entre SMTE et enseignant soit bonne et que leur collaboration soit efficace. Une idée sous-jacente à cette démarche est que la qualité de la collaboration entre les deux intervenants est influencée positivement par la connaissance réciproque de l'apport possible de chacun.

Dans cette optique, nous avons choisi d'orienter notre recherche vers une meilleure connaissance de la position des enseignants face aux services de support documentaire.

Comme élément de mesure, nous utiliserons la notion d'utilité. Après avoir consulté l'abondante documentation relative à la situation du SMTE dans l'enseignement ${ }^{2}$, nous avons opté pour ce critère en raison principalement de son caractère objectif: la notion d'utilité est facilement compréhensible, ce qui n'est pas le cas pour l'attitude par exemple. L'opinion des enseignants quant à l'utilité des services fournit une image concrète de la réalité; elle offre au SMTE des faits objectifs lui facilitant l'établissement, avec les enseignants, d'une communauté de pensée qui, bien qu'étrangère aux besoins véritables en ressources docu- mentaires, soit basée sur la connaissance des intentions du professeur.

\section{Les débats actuels}

La présente étude s'inscrit tout à fait dans le contexte des débats actuels. De nombreux auteurs ont traité de la collaboration entre SMTE et enseignants. Une tendance assez forte actuellement revendique pour le SMTE un élargissement de son rôle et l'inclusion des fonctions de conseiller pédagogique en ressources documentaires; ceci lui permettrait de participer, en étroite collaboration avec le professeur, à la préparation des cours et à l'élaboration des méthodes d'enseignement. Merril ${ }^{3}$ explique que cette conception n'est pas encore partagée par le personnel enseignant, que bibliothécaires et enseignants continuent de travailler indépendamment les uns des autres et que la bibliothèque n'est pas utilisée à son maximum.

La plupart des recherches actuelles tendent à faire la lumière sur un aspect ou l'autre des difficultés de cette intégration. Ainsi, s'interroge$t$-on sur la place du SMTE dans la vie pédagogique de l'institution et cherche-t-on à connaître la position des autres intervenants face à ce spécialiste ou au Centre de documentation. Chez les SMTE la description de leur rôle est loin de faire l'unanimité. Selon M. Jetter ${ }^{4}$, "the school media specialist of the future... will function as an instructionnal development specialist ". De même, selon Rosenberg 5 , «the media specialist is also a curriculum specialist » et, à ce titre, il doit rester en contact avec tous les aspects du programme scolaire, cours, objectifs des professeurs, programmes expérimentaux, etc.

Evidemment cette approche est contestée. On reconnaît que le SMTE doit être un intervenant important mais on ne sait toujours pas dans quelle mesure.

Ball6, en 1975, a recueilli l'opinion des SMTE sur cette question. En dépit de la constatation de
1. Le titre de spécialiste en moyens et techniques d'enseignement (SMTE) correspond à une classification administrative. II a été choisi pour identifier les professionnels de la documentation (écrite ou autre) et équivaut au «Library Media Specialist * américain.

2. De nombreuses recherches ont été et sont encore entreprises sur la situation des centres de documentation dans les maisons d'enseignement. Paulette Bernhard a recensé dans Dissertation Abstracts entre 1976 et 1982, 120 titres sur ce sujet en général (Argus, vol. 11, no 1 (janvier-février 1982), 22). C'est dire l'intérêt des chercheurs pour ce domaine.
3. M. Merril, Regular and Irregular Library Use by Faculty Members at Three Universities, thèse de doctorat, University of Pittsburg, 1979.

4. M.A. Jetter, The Roles of the School Library Media Specialist of the Future: a Delphi Study, thèse de doctorat, Michigan State University, 1972.

5. M. J. Rosenberg, $\propto$ What is the School Media Specialist's Role», Audio Visual Instruction, vol. 23 (February 1978), 12-13. 
leurs limitations et de leurs faiblesses, ils croient qu'ils devraient jouer un rôle central à l'intérieur du programme de l'établissement. Certains SMTE, comme E. Roe7, attirent l'attention sur le fait que ce spécialiste ne saurait en aucun cas se prévaloir d'un statut et d'un rôle qui ne serait pas accepté par les enseignants et les directeurs. Une idée fait cependant l'objet d'un consensus. Cette idée est bien exprimée par P. Knapp:

Neither subject field, nor teaching method, nor kind of assignment, nor quality of students in a class is of crucial importance in determining wether or not a given course will be dependent upon the library. The only decisive factor seemed to be - and this is a subjective judgement the instructor's attitude. Where the instructor expected and planned for student use of the library, it occured. Where he did not, it did not occur ${ }^{8}$.

On reconnaît donc le rôle primordial de l'enseignant. Toute cette réflexion sur le rôle du SMTE dans l'enseignement touche le coeur de la profession et a inspiré de nombreux sujets de recherche sur les facteurs affectant le comportement des enseignants face aux ressources humaines du centre de documentation. Shirley Blair dans une remarquable synthèse de vingt-quatre thèses de doctorat récentes a identifié huit facteurs de ce type:

The eight major factors identified as affecting teacher use of the school resource centre are cooperative curriculum planning; teacher inservice programs provided by the teacherlibrarian; administrative policies; physical facilities and tone and atmosphere of the resource centre; teacher attitudes, background and interests; and cooperative selection and evaluation of the collection?.

On constate que cinq facteurs sur huit ont trait aux relations entre SMTE et enseignants.

\section{Relations SMTE - enseignants}

Parmi toutes les approches proposées pour l'étude de la question de la collaboration entre
SMTE et enseignants, nous nous sommes particulièrement intéressé aux recherches orientées sur la mesure des attitudes face au centre de documentation ou face au SMTE, à celles basées sur la perception du rôle du SMTE par les divers intervenants, ainsi qu'à celles destinées à évaluer l'éventail et la qualité des services offerts.

Bien que ne générant pas de résultats susceptibles d'inspirer des actions concrètes aux praticiens, ces études nous sont apparues intéressantes en ce qu'elles illustrent la complexité du problème et la nature intangible des causes du succès ou de l'insuccès du SMTE dans son milieu.

Du côté des attitudes, R.K. Wood ${ }^{10}$ a montré que les attitudes des enseignants ne sont pas plus favorables après qu'avant un cours d'initiation à la bibliothèque. K. Ishikawa ${ }^{11}$ a démontré quant à lui l'existence d'une relation entre les services offerts et l'attitude des enseignants mais il n'a pas réussi à établir de liens entre les variables personnelles et professionnelles des enseignants et leur attitude. R. Smith 12, pour sa part, a identifié chez les enseignants des attitudes traditionnelles et d'autres plus contemporaines. C. Bish ${ }^{13} \mathrm{~s}^{\prime}$ est intéressé à l'attitude des enseignants face aux supports documentaires traditionnels et face aux supports nouveaux. II a constaté l'existence d'attitudes favorables face à l'ensemble des supports mais encore plus favorables aux supports traditionnels.

En ce qui touche la perception du rôle des SMTE, C.J. Anderson ${ }^{14}$ a montré que leur perception se rapproche davantage de celle des directeurs que de celle des enseignants. II a identifié de nombreuses divergences d'opinion entre enseignants et SMTE quant au rôle de ce dernier. P.F. Cantor ${ }^{15}$, dans une recherche semblable à celle d'Anderson, a mis l'accent sur la notion d'importance des différents rôles et il a constaté que $24 \%$ des chargés de classes affirment que les bibliothèques sont de la plus haute importance pour le succès de leur enseignement alors que $37 \%$ croient qu'elles ont une importance considérable.

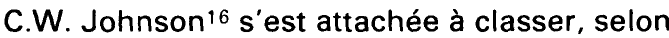
l'importance, différents domaines de compétence des SMTE à partir des opinions des différents
6. H. G. Ball, A Survey of the Issues, Problems and Concerns Affecting School Media Specialists in the Southern Parts of the United States, ERIC document, 1975.

7. M. Broadbent, «Differences of Opinions: the School Librarian's Role as Teachers see it", The Australian Library Journal, vol. 26 (September 1977), 253-256.

8. P. Knapp, "College Teaching and the Library», Illinois Libraries, vol. 40 (December 1958), 859.

9. S. Blair, «Teachers and the School Resource Centre», Canadian Library Journal, vol. 35, no. 2 (April 1978), 93-100.

10. R.K. Wood, A Study of Change in Secondary Teachers Attitudes Towards School Library Concepts After Instruction in Librarianship, thèse de maîtrise, Western Michigan University, 1969
11. K. Ishikawa, Teacher Attitudes towards School Library: An Investigation of Library Service Levels Related to Teachers Characteristics, thèse de doctorat, George Peabody College for Teachers, 1972.

12. R. Smith, Development of a Scale to Differentiate Traditional and Contemporary Attitudes Towards School Library Services, thèse de doctorat, The University of Florida, 1971.

13. C. Bish, A Study of Teachers' Attitudes towards Selected Technological Media of Instruction and Examination of Salient Variables Related to These Attitudes, thèse de doctorat, George Washington University, 1967.

14. C. J. Anderson, Role Expectation of the High-School Librarian as Perceived by Librarians, Principals and Teachers, thèse de doctorat, University of Oregon, 1970.

15. P.F. Cantor, Role Expectations for Library Media Specialists, School Administrators and Teachers, thèse de doctorat, Columbia University, 1975 
intervenants. Elle n'a pas trouvé de différence significative entre l'opinion des enseignants, celle des dirigeants et celle des SMTE. A.L. Hambleton ${ }^{17}$, dans une étude fondée en partie sur la mesure de la perception du rôle des SMTE, a noté que le consensus est faible chez ceux-ci quand on leur demande d'identifier les rôles jugés pertinents. Elle note aussi que ces spécialistes effectuent plus souvent des tâches reliées à la gestion des ressources documentaires que des tâches touchant la planification pédagogique.

Notons que la majorité des auteurs ont utilisé, pour comparer les opinions, des regroupements de services en catégories générales. En ce sens, nous avons trouvé très intéressante une étude de M.F. Macon ${ }^{18}$. Cette dernière explique les résultats observés sur le rôle des superviseurs de centres de documentation en présentant des différences non pas au niveau de catégories de rôles ou de services mais au niveau de chacun des éléments du questionnaire utilisé. Cette méthode nous semble plus facilement exploitable par le praticien et plus rigoureuse sur le plan méthodologique.

Les études portant sur les attitudes et sur le rôle des SMTE sont utiles si l'on veut connaître le contexte des relations SMTE-enseignants. Elles permettent aussi de dresser un inventaire des différents rôles, tâches ou services susceptibles d'évaluation. D'autres études, portant sur l'évaluation des centres de documentation, sont également utiles à cette fin. Ainsi les travaux de Loertscher ${ }^{19}$ et de Stroud ${ }^{20}$ fournissent des listes très intéressantes. Il en va de même de la recherche de N.W. Burt ${ }^{21}$. Ce dernier travail nous est apparu des plus pertinents. Utilisant un dérivé du «Purdue Self Evaluation System » développé par Stroud et Loertscher, Burt a soumis à des enseignants une liste de services à évaluer selon divers critères dont celui de «supportive». L'auteur a ainsi montré que les services dirigés vers les étudiants sont, en général, jugés plus «supportive» que les services de conseiller pédagogique en ressources documentaires destinés aux enseignants. Stroud, de son côté a noté que seulement un tiers des SMTE participent à des activités de planification pédagogique avec des enseignants.

\section{L'enquête}

Compte tenu de notre objectif, connaître l'opinion des enseignants quant à l'utilité des différents services de support documentaire, et nous inspirant des observations de certains auteurs, nous avons construit un instrument d'enquête répondant à deux critères: d'abord, qu'il présente tous les types de services offerts par le SMTE; ensuite, qu'il permette la comparaison entre des services à divers points de vue (public visé, support utilisé, rôle de conseiller pédagogique en ressources documentaires ou non). Une liste de 52 fonctions a été établie. Certaines ont été formulées de manière à ne différer entre elles que sur une dimension (ex.: professeur vs étudiant). Chaque fonction devait être évaluée selon une échelle en sept points allant de « tout à fait inutile" à "extrêmement utile, prioritaire» à la suite de la question suivante: Ce service est-il (utile-inutile) comme support documentaire à l'enseignement, à court ou à long terme? Les questionnaires ont été distribués à un échantillon de 314 enseignants de trois cégeps de la région de Montréal. L'échantillon a été établi en fonction de la spécialisation des enseignants. II est donc représentatif des trois secteurs d'enseignement: sciences humaines et sociales, sciences exactes, enseignement technique.

Cent soixante-dix-huit enseignants, soit $56 \%$ de l'échantillon, ont retourné leur questionnaire. Les données recueillies ont permis, dans un premier temps, de classer les 52 fonctions en ordre d'utilité. Les dix services suivants, présentés en ordre croissant d'utilité, ont été jugés les moins utiles par les enseignants

Participer au choix des manuels de base pour les cours;

Participer, en tant que partenaire du professeur, à la planification de son cours comme spécialiste en ressources documentaires;

Discuter avec un professeur des objectifs d'apprentissage de son cours;

Pour les besoins académiques, acheter des documents en évaluant soi-même ces besoins;

Proposer au professeur une méthode d'évaluation de son habileté à exploiter les ressources documentaires:

Elaborer à l'usage du professeur, une stratégie d'exploitation d'un film en classe;

Participer à la planification d'un cours lorsque les supports audiovisuels sont utilisés:

Mississippi, thèse de doctorat, Mississippi State University 1977.

19. D. Loertscher, Media Center Services to Teachers in Indiana Senior High-School, 1972-1973, Indiana University, 1973.

20. J. Stroud, Evaluation of Media Center Services by Media Staff, Teachers and Students in Indiana Middle and Junior High-Schools, thèse de doctorat, Purdue University, 1976.

21. N. W. Burt, The School Library Media Center in an Era of Change:Programs / Services seen as Supportive by Students, Teachers, Principal and Library Media Specialists, thèse de doctorat, University of Southern California, 1980. 
Organiser avec des étudiants des rencontres d'échange d'idées et d'expériences sur les ressources documentaires en enseignement;

Donner au professeur une opinion sur la valeur d'un document écrit dont il a recommandé I'achat;

Acheminer, aux membres du comité pédagogique, des suggestions pour l'élaboration de programmes tenant compte des ressources documentaires;

Élaborer pour les étudiants une grille d'évaluation des documents écrits;

Participer à titre de spécialiste en ressources documentaires aux travaux du comité pédagogique ou de son équivalent.

Les enseignants ont désigné les dix fonctions suivantes comme étant les plus utiles (ordre croissant d'utilité):

Guider le professeur qui cherche une information dans les ouvrages de référence;

Guider le professeur qui prépare un document audiovisuel pour son cours;

Rendre disponible en réserve des dossiers de textes pertinents à un cours;

Informer les étudiants sur l'évantail des services de support documentaire offerts;

Informer le professeur sur l'éventail des services de support documentaire offerts;

Guider l'étudiant qui cherche une information dans les ouvrages de référence;

Donner au professeur la liste des nouvelles acquisitions;

Pour les besoins académiques, acheter les documents demandés par les professeurs;

Fournir au professeur la liste des nouvelles parutions dans son domaine d'enseignement;

Initier les étudiants aux outils de repérage des documents: fichiers, index, bulletins signalétiques, ouvrages de référence, etc.

\section{Évaluation positive}

En général, les services de support documentaire à l'enseignement sont évalués positivement par les enseignants puisque les scores moyens varient de 3,48 à 6,26 sur l'échelle allant de 1 à 7 . Les analyses statistiques n'ont montré aucune divergence d'opinion entre les trois secteurs d'enseignement pour l'ensemble des 52 fonctions. Des différences significatives apparaissent cependant au niveau de certaines. En voici quelques exemples.

Les professeurs du secteur Sciences humaines et sociales jugent moins utiles que leurs confrères l'élaboration d'une grille d'évaluation de la valeur pédagogique des documents audiovisuels. Ceux du secteur des sciences exactes accordent peu d'importance à la fonction de mise au courant des nouveautés et à celle d'initiation à la manipulation des appareils audiovisuels. Par contre, ces mêmes personnes favorisent l'initiation des étudiants à l'utilisation de l'ordinateur comme support documentaire. De leur côté, les professeurs du secteur technique jugent utile qu'on fournisse au professeur la liste des nouvelles parutions dans son domaine d'enseignement, qu'on lui donne la liste des nouvelles acquisitions, qu'on l'informe des services de support documentaire disponibles, qu'on participe au choix des manuels de base pour les cours, qu'on initie les professeurs à l'utilisation des outils de repérage des documents.

Notons, au passage, qu'un seul de ces services s'adresse aux étudiants.

Certains éléments étaient formulés de manière à ce qu'on puisse les comparer entre eux. Vingtsix fonctions ont ainsi été appariées de façon à faciliter la comparaison de l'utilité selon les usagers, les professeurs ou les étudiants. Parmi les cas les plus intéressants notons les opinions suivantes: I'initiation aux outils de repérage des documents a été jugée plus utile pour les étudiants que pour les professeurs; on juge plus utile le travail destiné à corriger les lacunes des étudiants que celui visant des professeurs; on croit plus utile que le professeur soit informé des nouvelles acquisitions par rapport à l'étudiant.

Enfin, nous avons constaté que les questions, sept au total, portant sur l'utilité des services de conseiller pédagogique en documentation ont permis de situer ces services parmi les dix moins utiles.

Ces quelques données ne peuvent évidemment pas rendre compte adéquatement de l'opinion des enseignants. II est préférable, afin d'éviter les généralisations hâtives, d'examiner chacun des services dans le contexte et selon la formulation qui a été soumise aux enseignants. II reste que le simple examen des fonctions figurant en tête du classement selon le degré d'utilité est plutôt réconfortant. Ne serait-on pas heureux de satisfaire adéquatement les besoins jugés très utiles ou prioritaires pour les enseignants? Ne serions-nous pas en droit d'exiger les ressources nécessaires à la satisfaction de ces besoins essentiels?

Daniel Morin

École de bibliothéconomie

Université de Montréal

\section{ERRATA}

Une erreur d'impression s'est glissée dans l'article de Claude Martin intitulé "Les bibliothèques au Québec: une «industrie» de 150 millions \$» publié dans notre livraison d'avril-juin 1984. A la page 57,2 e col., 3 e paragraphe, 3 e ligne, on aurait dû lire: équivalentes à $6,5 \%$ de leurs dépenses d'opération (et non $65 \%$ ). 